\title{
Acute and Chronic Toxicity Pattern in Ketamine Abusers in Hong Kong
}

\author{
Yiu-Cheung Chan
}

Published online: 3 May 2012

(C) American College of Medical Toxicology 2012, corrected publication 2021

\begin{abstract}
Ketamine has been the commonest abusive substance used by Hong Kong teenager since 2005. It is also the fourth commonest poison encountered in Hong Kong Poison Information Centre (HKPIC) poisoning data in 2010. From June 2008 to July 2011, HKPIC managed 188 and 96 cases of acute and chronic ketamine poisoning, respectively, which reflect its acute and chronic toxicity pattern. Demographically, there is a male predominance, and the majority is between the ages of 10-39. For the acute cases, $48 \%$ presented with neurological features such as confusion, drowsiness, or transient loss of consciousness which usually subside with supportive care in a few hours. For the chronic cases, $92 \%$ of them presented with features of ketamine cystitis while about $66 \%$ presented with chronic abdominal pain. The current understanding of ketamine cystitis and chronic abdominal pain will be reviewed. Management is primarily symptomatic measures and most importantly abstinence from ketamine use.
\end{abstract}

Keyword Ketamine abuse

\section{Introduction}

Ketamine is a "dissociative anesthetic" that was first synthesized in 1964; it is used as an intravenous anesthetic therapeutically and has also increasingly been used as an

The original online version of this article was revised: Please note that author and surname were inadvertenly transposed in this article as original published: Chan Yui-Cheung should have read Yui-Cheung Chan

Y-C. Chan $(\bowtie)$

Hong Kong Poison Information Centre, K3A,

United Christian Hospital,

130 Hip Wo Street, Kwun Tong,

Hong Kong, SAR, People's Republic of China

e-mail: chanyc3@ha.org.hk abusive agent. In Hong Kong, ketamine has been the commonest abusive substance in teenagers since 2005 [1] according to the data of the Central Registry of Drug Abuse in which abusive cases are voluntarily reported by law enforcement departments, welfare agencies, health care facilities, and tertiary institutions. The Hong Kong Poison Information Centre (HKPIC) was established in 2005 and provides 24-h phone consultation service to health care professionals in Hong Kong for poison information and clinical management advice. It now receives about 4,500 poisoning cases per year. From the data of HKPIC, ketamine-related poisoning contributed to $7.1 \%$ of the poisoning case and is the fourth commonest poison encountered in the year 2010 [2]. Recreational ketamine abusers may seek medical advice for acute, sub-acute, or chronic presentations for physical, psychiatric, or social problems.

\section{Method}

From June 2008 to July 2011, the ketamine poisoning cases in the HKPIC database were retrieved to study the patterns of acute and chronic toxicity. Acute cases were defined as those who presented with an acute ketamine-related problem to the Accident and Emergency Department (AED) while chronic cases were those who presented with sub-acute or chronic problems in the outpatient clinic.

Data collected included patient demographic data (age, sex), exposure data (route, duration of ketamine abuse), clinical data (clinical features, investigations results), and management data (use of decontamination, antidotes, and other specific treatments). The presentation is further classified into each of the following entities clinically: neurological, urinary, cardiovascular, gastrointestinal, nasal, and psychiatric. 


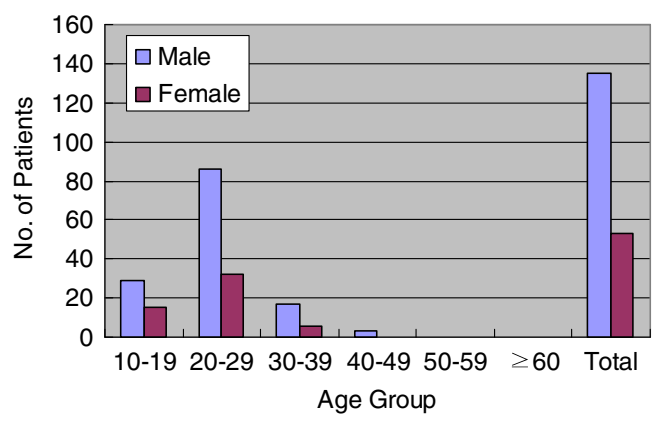

Fig. 1 Sex and age distribution of acute cases

\section{Results}

Totally, there were 284 cases, including 188 acute and 96 chronic cases, respectively. All cases abused ketamine by insufflation. The age and gender distribution of our cases was shown in Figs. 1 and 2.

For the 188 acute cases (Fig. 3), 90 cases (48\%) presented with neurological features such as confusion, drowsiness, or transient loss of consciousness which usually subsided with supportive care in 2 to $6 \mathrm{~h}$. Sixty cases $(32 \%)$ had lower urinary tract symptoms compatible with ketamine cystitis while $50(27 \%)$ abusers presented with cardiovascular features such as hypertension (systolic blood pressure $>160 \mathrm{mmHg}$ or diastolic pressure $>90 \mathrm{mmHg}$ ) and tachycardia (heart rate $>100$ per minute). Forty nine $(26 \%)$ of them presented with abdominal pain, and $19(10 \%)$ patients presented with acute psychiatric problems such as acute psychosis, respectively.

For the 96 chronic cases (Fig. 4), their mean and median duration of ketamine abuse is 8.6 years (SD, 4.1 years) and 4 years, respectively. Majority $(88,92 \%)$ of them presented with features of ketamine cystitis such as dysuria, urgency, and frequency while 63 (66\%), 40 (42\%), and 15 (16\%) of them presented with chronic abdominal pain, nasal problems (including three cases of septal perforation), and psychiatric features, respectively.

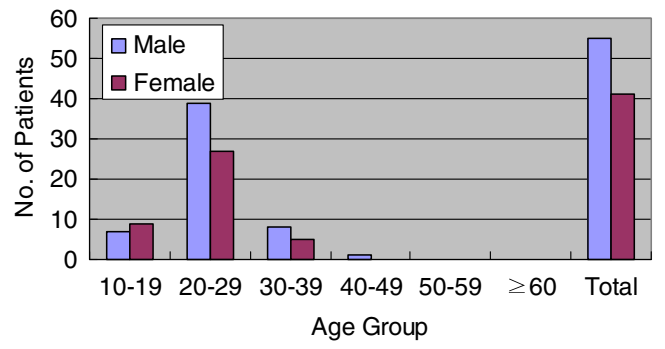

Fig. 2 Sex and age distribution of chronic cases

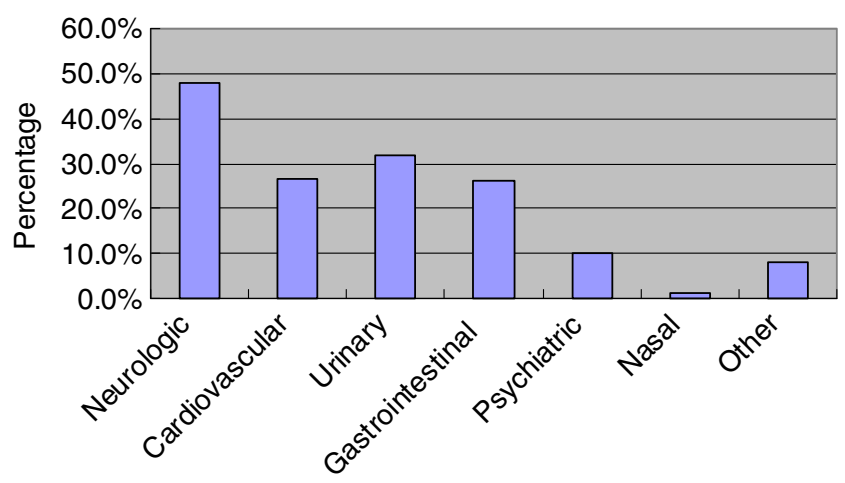

Fig. 3 Presenting symptoms of acute cases

\section{Discussion}

For the 188 acute cases, the commonest presentation was neurological features. The typical acute ketamine intoxication syndrome in our case series is confusion, dizziness, impaired conscious level, or a reported transient period of loss of consciousness, with the classical "nasal halo" (Fig. 5). From our clinical experience, nystagmus is also commonly seen on those cases who can comply with physical examination. About $27 \%$ of our cases had cardiovascular features; nearly all of them were in the form of mild hypertension and tachycardia. The clinical effect usually lasts for a few hours, and most cases can be managed with conservative and supportive measures. The findings are comparable to our study concerning the ketamine abusers' presentation in AED from 2005 to 2008 [3].

For the 96 chronic cases, $92 \%$ of them have features of ketamine cystitis. The typical clinical syndrome of ketamine cystitis includes lower urinary tract symptoms such as dysuria, urgency, and frequency. There are also features of small, painful bladder and upper urinary tract obstruction. Apart from Hong Kong, ketamine cystitis is known and

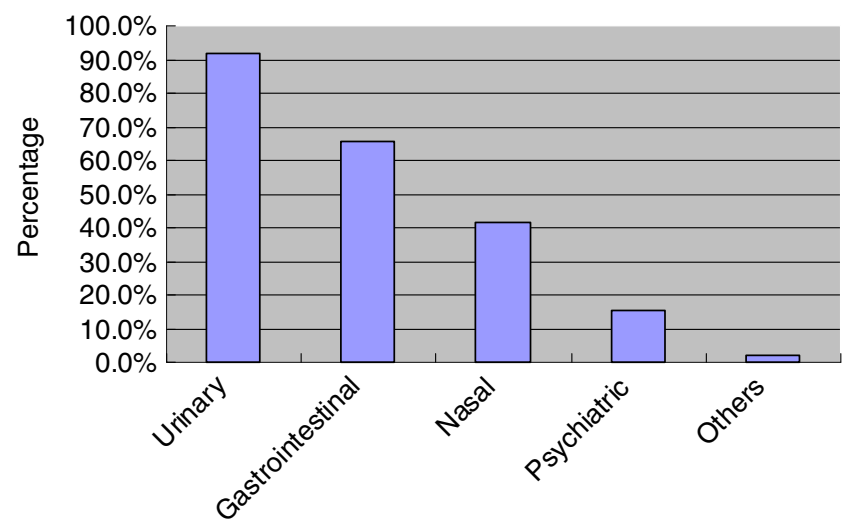

Fig. 4 Presenting symptoms of chronic cases 


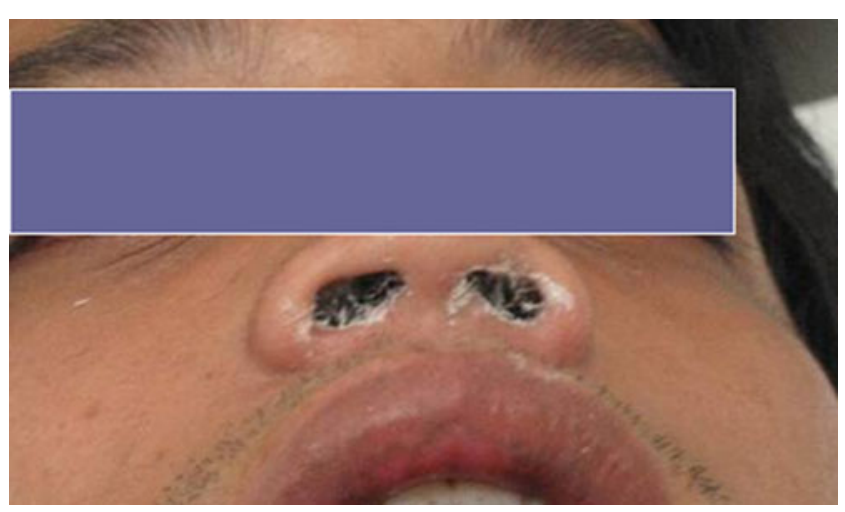

Fig. 5 Nasal halo

reported in countries including Canada, Japan, Taiwan, the $\mathrm{UK}$, and others $[4,5]$.

The causative agent for ketamine cystitis is believed to be ketamine itself or its metabolites. Although the possibility of unrecognized adulterants to be the cause for the syndrome is raised, it is very unlikely based on the fact that the syndrome is similar in different geographic areas over time in which the adulterant is expected to vary. For the mechanism of damage, it is still unconfirmed at this moment, and possible mechanisms including direct toxic damage, microvascular injury, or immune-related damage to the urinary tract by ketamine or/and its metabolites were suggested [6]. Concerning the duration of ketamine abuse and the development of ketamine cystitis, our data found the median years of exposure was 4 for our cohort in which $92 \%$ of them have features of ketamine cystitis. Another study in Hong Kong found that a ketamine abuser with at least 2 years of exposure (more than three uses per week) had significantly lower voided volume and higher scores in the Pelvic Pain, Urgency and Frequency questionnaire, which is comparable with our clinical experience [7].

Until now, no proven effective treatment for ketamine cystitis is established except ketamine abstinence. A recent study of 66 ketamine abusers found that the voided volume was significantly higher for those who quitted ketamine for more than 1 year than those who still abused ketamine or stopped it for less than 3 months [7].

In our chronic cases, two thirds had features of ketamineassociated abdominal pain. The pain is usually moderate to severe, dull or cramping pain in the epigastrium, with associated nausea and vomiting, and some cases have deranged liver function tests also. Case reports suggested the pain being biliary in origin [8-10], in the form of cholestasis and biliary dilatation with uniform pattern, with fusiform dilatation of the entire common hepatic and bile duct [8]. However, the mechanism of this pathology remains unknown. In a retrospective study of 37 ketamine abusers for the upper gastrointestinal problems [11], $75.7 \%$ of them had upper GI symptoms, which are comparable to our chronic cohort. Fifteen $(40.5 \%)$ and $9(24.3 \%)$ of them had elevated alanine aminotransferase (ALT) and alkaline phosphate (ALP), respectively, but the study was unable to demonstrate any association between epigastric pain and deranged ALT or ALP. Only 14 of the abusers underwent an esophagogastroduodenoscopy examination in which 12 of them had gastritis while one abuser had gastroduodenitis and the other had a normal finding.

The management of ketamine-associated abdominal pain is primarily symptomatic and again, most importantly, abstinence from ketamine use. It was shown that the odd ratio of symptomatic relief of the abdominal pain is 12.5 (95\% CI 1.2-130) for those who stopped ketamine versus those who continued its use [11].

There are several limitations of this study. First of all, our consultation and reporting system was on a completely voluntary basis. Secondly, data were obtained from the consulting medical professions and may be incomplete in some case. However, given the number of case we received (4,500 per year) and the accessibility to the clinical records of nearly all of our consultation/reporting cases, we believed the data represented the updated acute and chronic presentations of ketamine abusers in Hong Kong.

\section{Conclusion}

We present a large case series of 284 ketamine abuser with regard to their acute and chronic toxic manifestations. In acute intoxication, neurological features in terms of confusion and impaired consciousness are the commonest presentations. For chronic presentations, majority had features of ketamine cystitis and chronic abdominal pain. Management is primarily symptomatic measures and most importantly abstinence from ketamine use.

\section{References}

1. Narcotics Division, Security Bureau, the Government of Hong Kong Special Administrative Region of the People's Republic of China (2009) Central registry of drug abuse, http://www.nd.gov.hk/en/drug statistics.htm. Accessed on 13 Feb 2012

2. Chan YC, Tse ML, Lau FL (2012) Hong Kong Poison Information Centre: annual report 2010. Hong Kong J Emerg Med 19:110

3. Ng SH, Tse ML, Ng HW, Lau FL (2010) Emergency department presentation of ketamine abusers in Hong Kong: a review of 233 cases. Hong Kong Med J 16(1):6-11

4. Wood D, Cottrell A, Baker SC, Southgate J, Harris M, Fulford S, Woodhouse C, Gillatt D (2011) Recreational ketamine: from pleasure to pain. BJU Int 107(12):1881-1884

5. Nomiya A, Nishimatsu H, Homma Y (2011) Interstitial cystitis symptoms associated with ketamine abuse: the first Japanese case. Int J Urol 18(10):735 
6. Chu PS, Kwok SC, Lam KM, Chu TY, Chan SW, Man CW, Ma WK, Chui KL, Yiu MK, Chan YC, Tse ML, Lau FL (2007) 'Street ketamine'-associated bladder dysfunction: a report of ten cases. Hong Kong Med J 13(4):311-313

7. Mak SK, Chan MT, Bower WF, Yip SK, Hou SS, Wu BB, Man CY (2011) Lower urinary tract changes in young adults using ketamine. J Urol 186(2):610-614

8. Wong SW, Lee KF, Wong J, Ng WW, Cheung YS, Lai PB (2009) Dilated common bile ducts mimicking choledochal cysts in ketamine abusers. Hong Kong Med J 15(1):53-56
9. Lo RS, Krishnamoorthy R, Freeman JG, Austin AS (2011) Cholestasis and biliary dilatation associated with chronic ketamine abuse: a case series. Singap Med J 52(3):e52e55

10. Seto WK, Ng M, Chan P, Ng IO, Cheung SC, Hung IF, Yuen MF, Lai CL (2011) Ketamine-induced cholangiopathy: a case report. Am J Gastroenterol 106(5):1004-1005

11. Poon TL, Wong KF, Chan MY, Fung KW, Chu SK, Man CW, Yiu MK, Leung SK (2010) Upper gastrointestinal problems in inhalational ketamine abusers. J Dig Dis 11(2):106-110 